\title{
Simultaneous calculation of the helical pitch and the twist elastic constant in chiral liquid crystals from intermolecular torques
}

\author{
Guido Germanoa) \\ Theoretische Physik, Universität Bielefeld, 33501 Bielefeld, Germany \\ Michael P. Allen ${ }^{\text {b) }}$ \\ Centre for Scientific Computing and Department of Physics, University of Warwick, Coventry CV4 7AL, \\ United Kingdom \\ Andrew J. Masters ${ }^{\mathrm{c})}$ \\ Department of Chemistry, University of Manchester, Oxford Road, Manchester M13 9PL, United Kingdom
}

(Received 2 January 2002; accepted 12 March 2002)

\begin{abstract}
We present a molecular simulation method that yields simultaneously the equilibrium pitch wave number $q$ and the twist elastic constant $K_{2}$ of a chiral nematic liquid crystal by sampling the torque density. A simulation of an untwisted system in periodic boundary conditions gives the product $K_{2} q$; a further simulation with a uniform twist applied provides enough information to separately determine the two factors. We test our new method for a model potential, comparing the results with $K_{2} q$ from a thermodynamic integration route, and with $K_{2}$ from an order fluctuation analysis. We also present a thermodynamic perturbation theory analysis valid in the limit of weak chirality. (C) 2002 American Institute of Physics. [DOI: 10.1063/1.1475747]
\end{abstract}

\section{INTRODUCTION}

Chirality is of paramount importance in chemistry as well as in liquid crystal (LC) display technology, where devices are often based on the interaction of chiral or twisted nematic LCs with polarized light having the same wavelength as their pitch $P$, that is the distance over which the director rotates through an angle $2 \pi .{ }^{1}$ Therefore, there is an understandable interest in forecasting the pitch of mesophases, be they pure liquids of chiral molecules, or solutions of chiral dopants in achiral solvents, from a knowledge of their molecular structures. In a molecular simulation, it is currently impractical to realize the equilibrium pitch for a given system: the length and time scales are too long. However, the pitch is determined by phenomenological coefficients in the elastic free energy, and these coefficients may be obtained by other methods. When a finite pitch is imposed externally, for instance by the condition of compatibility with periodic boundary conditions (PBCs), ${ }^{2}$ a free energy difference arises between otherwise equivalent systems of mirrorimage molecules. By computing the chemical potential difference between right- and left-handed forms of a dopant molecule, for instance, the helical twisting power may be deduced. This approach was first proposed for the case where the dopant is a dimer, essentially composed of two solvent molecules in a particular conformation: ${ }^{3,4}$ in this case, the chemical potential difference may be calculated with good statistics. Later, this quantity has been calculated by a more conventional approach, namely thermodynamic integration: one enantiomer is slowly mutated into the other in a step-bystep calculation. ${ }^{5}$ This removes the restriction to dimers, but

\footnotetext{
${ }^{a)}$ Electronic mail: germano@physik.uni-bielefeld.de

${ }^{b)}$ Electronic mail: m.p.allen@warwick.ac.uk

c)Electronic mail: andrew.masters@man.ac.uk
}

the technique is computationally expensive, and care must be taken: the quantity of interest is very small in comparison with the absolute values calculated in the simulation. By whatever means the appropriate term in the free energy is obtained, it may be shown to reduce to a product $h=K_{2} q$ of the twist elastic constant $K_{2}$ and the wave number $q$ $=2 \pi / P$ characterizing the equilibrium pitch $P$.

In Sec. II we propose a new method that is also quite general, but simpler, since it does not require a mutation of the system. Instead, the results come from equilibrium torque measurements in runs with conventional and twisted PBCs; moreover, the results may be combined to yield $K_{2}$ and $q$ separately. In Sec. III we describe the model potential and the simulation procedure used to test the method. The results are presented in Sec. IV, and in the final discussion in Sec. V we conclude this paper.

\section{THEORY}

For notational convenience we assume that the total potential energy of the system, denoted $\mathcal{U}_{\lambda}$, may be written so as to depend on a chirality parameter $\lambda$; that changing the sign of $\lambda$ gives an enantiomeric system, i.e., one with the opposite sign of equilibrium pitch wave number, which we denote $q_{\lambda}$; and that $\lambda=0$ corresponds to a molecular model that is achiral by symmetry. Later, we shall consider the case in which $\lambda$ measures the strength of a chiral perturbation term in the potential, paying attention to the limit of small $\lambda$, but we do not make that assumption at this stage: $\lambda$ might represent some internal parameter defining the molecular structure, for example, a twist angle about some bond, which is not necessarily small. More generally, $\lambda$ may be a thermodynamic integration parameter.

The Frank elastic free energy for a chiral nematic (cholesteric) phase with equilibrium pitch $q_{\lambda}$ is ${ }^{1}$ 


$$
\begin{aligned}
\mathcal{F}= & \frac{1}{2} \int\left\{K_{1}(\boldsymbol{\nabla} \cdot \hat{\mathbf{n}})^{2}+K_{2}\left[\hat{\mathbf{n}} \cdot(\boldsymbol{\nabla} \times \hat{\mathbf{n}})+q_{\lambda}\right]^{2}\right. \\
& \left.+K_{3}[\hat{\mathbf{n}} \times(\boldsymbol{\nabla} \times \hat{\mathbf{n}})]^{2}\right\} \mathrm{d} \mathbf{r} ;
\end{aligned}
$$

$K_{1}, K_{2}$, and $K_{3}$ are the splay, twist, and bend elastic constants; the unit vector $\hat{\mathbf{n}}(\mathbf{r})$ represents the director field. Inserting the expression for the director of a phase uniformly twisted along the $z$ axis,

$$
\begin{aligned}
& \hat{\mathbf{n}}(\mathbf{r})=[\cos \phi(z), \sin \phi(z), 0], \\
& \frac{\mathrm{d} \phi(z)}{\mathrm{d} z}=k=\mathrm{const},
\end{aligned}
$$

and integrating over the volume $V$, we get

$$
\mathcal{F}_{k, \lambda}=\frac{1}{2} V K_{2}\left(k-q_{\lambda}\right)^{2},
$$

where the subscripts remind us of the dependence on both the twist wave number $k$ and the chirality strength $\lambda$. The reference state of minimum (here zero) free energy has $k$ $=q_{\lambda}$; nematics are called chiral if $q_{\lambda} \neq 0$ and twisted if $k$ $\neq q_{\lambda}$. Uniform twists with $k \neq q_{\lambda}$ are due, typically, to boundary conditions produced by the interaction with surfaces. However, in computer simulations the same effect can be introduced in a bulk cell of fluid through the influence of the PBCs. In conventional, untwisted, PBCs, periodicity restricts the helical pitch such that an integer number of halfturns take place in one box length. This means that

$$
\frac{P}{L_{z}}=\frac{2}{n_{z}}, \quad k L_{z}=2 \pi \frac{L_{z}}{P}=n_{z} \pi,
$$

where $L_{z}$ is the length of the simulation box and $n_{z}$ is an integer. The state with $k$ closest to $q_{\lambda}$ has the lowest free energy, but states with nearby values of $n_{z}$ are metastable: there is a free energy barrier between states of different $n_{z}$. In twisted PBCs, ${ }^{2}$ the coordinates and orientations of image molecules in the neighboring simulation boxes along the $\pm z$ direction are rotated by a quarter-turn, $\pm \pi / 2$, with respect to their values in the reference box. In this case, the pitch may take the values

$$
\frac{P}{L_{z}}=\frac{2}{n_{z}+\frac{1}{2}}, \quad k L_{z}=2 \pi \frac{L_{z}}{P}=\left(n_{z}+\frac{1}{2}\right) \pi .
$$

It should be borne in mind that in computer simulations, if we were to use a molecular model with a realistic degree of chirality, the state closest to $q_{\lambda}$ would generally be $k=0$, because the accessible length scales in simulations are substantially shorter than the natural equilibrium pitches seen experimentally.

\section{A. Thermodynamic integration method}

Using Eq. (3), the free energy difference between two enantiomeric phases, that have equilibrium twist wave numbers $q_{ \pm \lambda}= \pm q_{\lambda}$ of opposite sign, is

$$
\begin{aligned}
\Delta \mathcal{F}_{k, \pm \lambda} & \equiv \mathcal{F}_{k, \lambda}-\mathcal{F}_{k ;-\lambda} \\
& =\frac{1}{2} V K_{2}\left[\left(k-q_{\lambda}\right)^{2}-\left(k+q_{\lambda}\right)^{2}\right] \\
& =-2 V K_{2} q_{\lambda} k .
\end{aligned}
$$

This free energy difference may be computed in a simulation by thermodynamic integration along a path that mutates all molecules into their mirror-image forms, while maintaining the system in a state of constant nonzero $k$ through the chosen PBCs. Hence, such a technique will yield the product $h_{\lambda} \equiv K_{2} q_{\lambda}$, and the value of $q_{\lambda}$ may be deduced from a separate calculation of $K_{2}$ (which may also depend on $\lambda$, but we suppress this in the notation, anticipating that the dependence will be higher order than linear). Assuming that we need only consider the excess (nonideal) contribution to $\mathcal{F}$, the explicit formula follows from the statistical mechanical relation

$$
\frac{\partial \mathcal{F}_{k, \lambda}}{\partial \lambda}=\left\langle\frac{\partial \mathcal{U}_{\lambda}}{\partial \lambda}\right\rangle_{k, \lambda},
$$

which underpins thermodynamic perturbation theory. ${ }^{6,7}$ This gives the free energy difference between two enantiomeric phases at a specified twist wave number $k$,

$$
\begin{aligned}
\Delta \mathcal{F}_{k, \pm \lambda} & =\int_{-\lambda}^{\lambda} \frac{\partial \mathcal{F}_{k, \lambda^{\prime}}}{\partial \lambda^{\prime}} \mathrm{d} \lambda^{\prime} \\
& =\int_{-\lambda}^{\lambda}\left\langle\left.\frac{\partial \mathcal{U}_{\lambda^{\prime}}}{\partial \lambda^{\prime}}\right|_{k, \lambda^{\prime}} \mathrm{d} \lambda^{\prime}\right. \\
& \equiv \int_{-\lambda}^{\lambda}\left\langle\mathcal{U}_{\lambda^{\prime}}^{c}\right\rangle_{k, \lambda^{\prime}} \mathrm{d} \lambda^{\prime},
\end{aligned}
$$

where $\langle\cdots\rangle_{k, \lambda^{\prime}}$ represents a simulation average conducted at chirality $\lambda^{\prime}$, in boundaries that impose a twist wave number $k$, and we have defined

$$
\mathcal{U}_{\lambda}^{c} \equiv \frac{\partial \mathcal{U}_{\lambda}}{\partial \lambda}
$$

Hence

$$
h_{\lambda} \equiv K_{2} q_{\lambda}=-\frac{1}{2 V k} \int_{-\lambda}^{\lambda}\left\langle\mathcal{U}_{\lambda^{\prime}}^{c}\right\rangle_{k, \lambda^{\prime}} \mathrm{d} \lambda^{\prime} .
$$

Thus, we may approach the calculation of the helical pitch in the standard framework of thermodynamic linear response theory, but we rely on the explicit calculation of the appropriate potential energy derivative with respect to $\lambda$, Eq. (6).

\section{B. Torque measurement method}

The method to be examined here exploits the microscopic expression for the torque per unit area. ${ }^{2}$ Begin by defining the tensor 


$$
\begin{aligned}
\Pi_{\alpha \beta} & =-\sum_{i} r_{i \alpha} \tau_{i \beta} \\
& =-\frac{1}{2} \sum_{i \neq j} r_{i j \alpha} \tau_{i j \beta} \\
& =-\frac{1}{2} \sum_{i<j} r_{i j \alpha}\left(\tau_{i j \beta}-\tau_{j i \beta}\right),
\end{aligned}
$$

with $\alpha, \beta=x, y, z ; r_{i j \alpha}=r_{i \alpha}-r_{j \alpha}$ is the $\alpha$ component of the separation of the centers of mass; $\tau_{i \beta}$ is the $\beta$ component of the torque acting on molecule $i$,

$$
\boldsymbol{\tau}_{i}=-\hat{\mathbf{e}}_{i} \times \frac{\partial}{\partial \hat{\mathbf{e}}_{i}} \mathcal{U}_{\lambda},
$$

where $\hat{\mathbf{e}}_{i}$ is a unit vector along the main molecular axis (the molecule may not be uniaxial). In Eq. (8) we have assumed that $\boldsymbol{\tau}_{i}$ can be expressed in pairwise contributions $\boldsymbol{\tau}_{i j}$ from all other molecules $j ; \boldsymbol{\tau}_{j i}$ is the corresponding torque exerted on $j$ by $i$. With the twist axis chosen along $z$, we shall be interested in the component $\Pi_{z z}$, which we denote $\Pi_{\lambda}$ henceforth to highlight the explicit dependence on $\lambda$, while maintaining notational simplicity. The torque per unit area may then be expressed microscopically: ${ }^{2}$

$$
\frac{\partial \mathcal{F}_{k, \lambda}}{\partial k}=\left\langle\Pi_{\lambda}\right\rangle_{k, \lambda}
$$

A comparison of this equation with the macroscopic expression $^{1}$

$$
\frac{1}{V} \frac{\partial \mathcal{F}_{k, \lambda}}{\partial k}=K_{2}\left(k-q_{\lambda}\right),
$$

once with $k=0$ and once with $k \neq 0$, gives the equations

$$
\begin{gathered}
h_{\lambda} \equiv K_{2} q_{\lambda}=-\frac{\left\langle\Pi_{\lambda}\right\rangle_{0, \lambda}}{V}, \\
K_{2}\left(q_{\lambda}-k\right)=-\frac{\left\langle\Pi_{\lambda}\right\rangle_{k, \lambda}}{V},
\end{gathered}
$$

and hence

$$
\begin{gathered}
K_{2}=\frac{\left\langle\Pi_{\lambda}\right\rangle_{k, \lambda}-\left\langle\Pi_{\lambda}\right\rangle_{0, \lambda}}{V k}=\left.\frac{1}{V} \frac{\partial\left\langle\Pi_{\lambda}\right\rangle_{k, \lambda}}{\partial k}\right|_{0, \lambda}, \\
q_{\lambda}=\frac{-k\left\langle\Pi_{\lambda}\right\rangle_{0, \lambda}}{\left\langle\Pi_{\lambda}\right\rangle_{k, \lambda}-\left\langle\Pi_{\lambda}\right\rangle_{0, \lambda}}=\frac{-\left\langle\Pi_{\lambda}\right\rangle_{0, \lambda}}{\partial\left\langle\Pi_{\lambda}\right\rangle_{k, \lambda} /\left.\partial k\right|_{0, \lambda}} .
\end{gathered}
$$

Thus, for molecules of a given chirality $\lambda$, two simulations with different values of $k$ are sufficient to determine $K_{2}$ and $q_{\lambda}$ separately: this is one of the principal results of this paper. Note that in Eqs. (12)-(15), it is not necessary to write $\mathcal{U}_{\lambda}$ or $\Pi_{\lambda}$ explicitly as a function of $\lambda$, as no thermodynamic integration with respect to a chirality parameter is used. The same formulas hold equally well for pure phases, where every molecule is chiral, and solutions, where chiral and achiral molecules are mixed. Equation (14) has previously been used $^{2}$ to calculate $K_{2}$ in fluids of achiral molecules, for which $\left\langle\Pi_{0}\right\rangle_{0,0}=0$ but $\left\langle\Pi_{0}\right\rangle_{k, 0} \neq 0$; and Eq. (12) has recently been proposed ${ }^{8-10}$ as a route to $h_{\lambda}$ for chiral molecules, for which $\left\langle\Pi_{\lambda}\right\rangle_{0, \lambda} \neq 0$. We note in passing that the tensor $\Pi_{\alpha \beta}$ was first introduced in expressions for the flexoelectric coefficients of pear- and banana-shaped mesogens, ${ }^{11}$ and that these expressions have recently been used in simulations. ${ }^{12}$

We emphasize that $\Pi$ is defined in terms of internal molecule-molecule torques, not the torques applied to the system at the boundaries, which may be necessary to maintain a twisted nematic state. The link with the externally applied torque per unit area is made by analogy with the derivation of the usual virial expression for the pressure. ${ }^{7,13}$ Consider the relation

$$
\left\langle\frac{\partial}{\partial t} \sum_{i} r_{i \alpha} j_{i \beta}\right\rangle=\left\langle\sum_{i} v_{i \alpha} j_{i \beta}\right\rangle+\left\langle\sum_{i} r_{i \alpha} \tau_{i \beta}^{\mathrm{tot}}\right\rangle=0,
$$

where $j_{i \beta}$ is a component of the intrinsic angular momentum of molecule $i$ and $\tau_{i \beta}^{\text {tot }}=\tau_{i \beta}+\tau_{i \beta}^{\text {ext }}$ is the total torque on the molecule due to both internal and external (boundary) effects. Separating these two contributions, and noting that $v_{i \alpha}$ and $j_{i \beta}$ are uncorrelated, gives

$$
\left\langle\Pi_{\alpha \beta}\right\rangle=\left\langle\sum_{i} r_{i \alpha} \tau_{i \beta}^{\mathrm{ext}}\right\rangle .
$$

This equation applies regardless of the degree of twist, or the molecular chirality. The expression on the right (divided by $V)$ is the macroscopic torque per unit area applied to the system; the expression on the left is entirely in terms of internal torques.

\section{Weak chirality}

The expressions of the previous section hold for arbitrary values of chirality strength $\lambda$. It will prove useful to consider the case of weak chirality, anticipating linear dependence on $\lambda$ of $q_{\lambda}$ and $h_{\lambda} \equiv K_{2} q_{\lambda}$ :

$$
q_{\lambda}=q^{\prime} \lambda, \quad h_{\lambda}=h^{\prime} \lambda,
$$

where $q^{\prime}$ and $h^{\prime}$ are the proportionality constants to be determined. The free energy relations of interest to us, at small $\lambda$ and $k$, are

$$
\begin{aligned}
& \left.\frac{1}{V} \frac{\partial \mathcal{F}_{k, \lambda}}{\partial \lambda}\right|_{k, 0}=-h^{\prime} k, \\
& \left.\frac{1}{V} \frac{\partial \mathcal{F}_{k, \lambda}}{\partial k}\right|_{0, \lambda}=-h^{\prime} \lambda, \\
& \left.\frac{1}{V} \frac{\partial^{2} \mathcal{F}_{k, \lambda}}{\partial \lambda \partial k}\right|_{0,0}=-h^{\prime} .
\end{aligned}
$$

We apply thermodynamic linear response theory, ${ }^{6,7}$ restricting our interest to the class of models for which the total intermolecular (internal) potential energy of the system, $\mathcal{U}_{\lambda}$, may be decomposed into an achiral part $\mathcal{U}^{a}$ and a $\lambda$-independent chiral term $\mathcal{U}^{c}$ multiplied by a strength parameter $\lambda$ :

$$
\mathcal{U}_{\lambda}=\mathcal{U}^{a}+\lambda \mathcal{U}^{c}
$$

each term is assumed, for convenience, to be pairwise additive. From the definition (8), we may decompose $\Pi_{\lambda}$ into achiral and chiral parts, in the same way: 


$$
\Pi_{\lambda}=\Pi^{a}+\lambda \Pi^{c} .
$$

We must also carefully consider the imposed twist, generated in a simulation by using the boundary conditions described earlier. For the analysis presented here, it is convenient to consider that this twist is produced by applying an external potential, so $\mathcal{U}^{\text {tot }}=\mathcal{U}+\mathcal{U}^{\text {ext }}$. The pitch wave number $k$ is determined by $\mathcal{U}^{\text {ext }}$, and we assume that we may divide it, like $\mathcal{U}$, into chiral and achiral parts: $\mathcal{U}_{k, \lambda}^{\text {ext }}=\mathcal{U}_{k}^{\text {ext, }, a}+\lambda \mathcal{U}_{k}^{\text {ext, },}$. In carrying out linear response theory it is important to include both the internal and external potentials.

Now all the $\lambda$ dependence is explicit; for small $\lambda$ we have $e^{6,7}$

$$
\begin{aligned}
\mathcal{F}_{k, \lambda}= & \mathcal{F}_{k, 0}+\lambda\left\langle\mathcal{U}^{\mathrm{tot}, c}\right\rangle_{k, 0}-\frac{1}{2} \lambda^{2} \beta\left(\left\langle\mathcal{U}^{\mathrm{tot}, c^{2}}\right\rangle_{k, 0}-\left\langle\mathcal{U}^{\mathrm{tot}, c}\right\rangle_{k, 0}^{2}\right) \\
+ & \cdots, \\
\left\langle\mathcal{U}^{a}\right\rangle_{k, \lambda}= & \left\langle\mathcal{U}^{a}\right\rangle_{k, 0}-\lambda \beta\left(\left\langle\mathcal{U}^{a} \mathcal{U}^{\mathrm{tot}, c}\right\rangle_{k, 0}-\left\langle\mathcal{U}^{a}\right\rangle_{k, 0}\left\langle\mathcal{U}^{\mathrm{tot}, c}\right\rangle_{k, 0}\right) \\
& +\cdots, \\
\left\langle\mathcal{U}^{c}\right\rangle_{k, \lambda}= & \left\langle\mathcal{U}^{c}\right\rangle_{k, 0}-\lambda \beta\left(\left\langle\mathcal{U}^{c} \mathcal{U}^{\mathrm{tot}, c}\right\rangle_{k, 0}-\left\langle\mathcal{U}^{c}\right\rangle_{k, 0}\left\langle\mathcal{U}^{\mathrm{tot}, c}\right\rangle_{k, 0}\right) \\
& +\cdots, \\
\left\langle\Pi^{a}\right\rangle_{k, \lambda}= & \left\langle\Pi^{a}\right\rangle_{k, 0}-\lambda \beta\left(\left\langle\Pi^{a} \mathcal{U}^{\mathrm{tot}, c}\right\rangle_{k, 0}-\left\langle\Pi^{a}\right\rangle_{k, 0}\left\langle\mathcal{U}^{\mathrm{tot}, c}\right\rangle_{k, 0}\right) \\
& +\cdots, \\
\left\langle\Pi^{c}\right\rangle_{k, \lambda}= & \left\langle\Pi^{c}\right\rangle_{k, 0}-\lambda \beta\left(\left\langle\Pi^{c} \mathcal{U}^{\mathrm{tot}, c}\right\rangle_{k, 0}-\left\langle\Pi^{c}\right\rangle_{k, 0}\left\langle\mathcal{U}^{\mathrm{tot}, c}\right\rangle_{k, 0}\right) \\
& +\cdots .
\end{aligned}
$$

The $k, 0$ subscript refers to an average carried out at $\lambda=0$ and in the presence of an external potential $\mathcal{U}_{k}^{\mathrm{ext}, a}$, i.e., the external potential that produces the desired twist in a system of purely achiral molecules. Clearly $\mathcal{U}_{k}^{\text {ext, } a}=0$ when $k=0$, as a system of achiral molecules is already untwisted in the absence of external potentials.

Thus, we expect to see a linear dependence of measurable properties on $\lambda$ : the zeroth-order term is related to the average values for achiral molecules under the same conditions of twist, while the first-order term reflects equilibrium correlations with $\mathcal{U}^{\text {tot, } c}$ in the $\lambda=0$ limit. From Eqs. (5) and (18c) we obtain

$$
\begin{aligned}
& \left.\frac{\partial \mathcal{F}_{k, \lambda}}{\partial \lambda}\right|_{k, 0}=\left\langle\mathcal{U}^{\mathrm{tot}, c}\right\rangle_{k, 0} \\
& \Rightarrow h^{\prime}=-\left.\frac{1}{V} \frac{\partial^{2} \mathcal{F}_{k, \lambda}}{\partial k \partial \lambda}\right|_{0,0}=-\left.\frac{1}{V} \frac{\partial\left\langle\mathcal{U}^{\mathrm{tot}, c}\right\rangle_{k, 0}}{\partial k}\right|_{0,0} .
\end{aligned}
$$

With some care ${ }^{14}$ this may be converted into a fluctuation expression valid at $k=0$ :

$$
\begin{aligned}
h^{\prime} & =-\frac{1}{V}\left[\left\langle\Pi^{c}\right\rangle_{0,0}-\beta\left(\left\langle\Pi_{\lambda} \mathcal{U}^{\mathrm{tot}, c}\right\rangle_{0,0}-\left\langle\Pi_{\lambda}\right\rangle_{0,0}\left\langle\mathcal{U}^{c}\right\rangle_{0,0}\right)\right] \\
& =-\frac{1}{V}\left[\left\langle\Pi^{c}\right\rangle_{0,0}-\beta\left\langle\Pi^{a} \mathcal{U}^{\mathrm{tot}, c}\right\rangle_{0,0}\right],
\end{aligned}
$$

where we use $\left\langle\mathcal{U}^{\text {tot,c }}\right\rangle_{0,0}=0$ (see the Appendix). An alternative derivation starts from Eq. (10):

$$
\begin{aligned}
\left.\frac{\partial \mathcal{F}_{k, \lambda}}{\partial k}\right|_{0, \lambda} & =\left\langle\Pi_{\lambda}\right\rangle_{0, \lambda} \\
\Rightarrow h^{\prime} & =-\left.\frac{1}{V} \frac{\partial^{2} \mathcal{F}_{k, \lambda}}{\partial \lambda \partial k}\right|_{0,0}=-\left.\frac{1}{V} \frac{\partial\left\langle\Pi_{\lambda}\right\rangle_{0, \lambda}}{\partial \lambda}\right|_{0,0} .
\end{aligned}
$$

This last equation may be converted into a fluctuation average as well:

$$
\begin{aligned}
h^{\prime}= & -\frac{1}{V}\left[\left\langle\frac{\partial \Pi_{\lambda}}{\partial \lambda}\right\rangle_{0,0}-\beta\left(\left\langle\Pi_{\lambda} \mathcal{U}^{\mathrm{tot}, c}\right\rangle_{0,0}\right.\right. \\
& \left.\left.-\left\langle\Pi_{\lambda}\right\rangle_{0,0}\left\langle\mathcal{U}^{\mathrm{tot}, c}\right\rangle_{0,0}\right)\right] \\
= & -\frac{1}{V}\left[\left\langle\Pi^{c}\right\rangle_{0,0}-\beta\left\langle\Pi^{a} \mathcal{U}^{\mathrm{tot}, c}\right\rangle_{0,0}\right],
\end{aligned}
$$

giving the same result as Eq. (27). This equation may be simplified by making use of the hypervirial theorem; thus

$$
-\beta\left\langle\Pi_{\alpha \beta}^{a} \mathcal{U}^{\mathrm{tot}, c}\right\rangle_{0,0}=\sum_{i}\left\langle r_{i \alpha}\left(\tau_{i \beta}^{c}+\tau_{i \beta}^{\mathrm{ext}, c}\right)\right\rangle_{0,0}=0 .
$$

The final equality is obtained by using Eq. (16), where the averages are evaluated at $k=0$ but at nonzero $\lambda$. A first-order expansion in powers of $\lambda$ gives the required result. Hence, we arrive at

$$
h^{\prime}=\frac{h_{\lambda}}{\lambda}=-\frac{\left\langle\Pi^{c}\right\rangle_{0,0}}{V} .
$$

Equation (14), evaluated in the limit $\lambda \rightarrow 0$, becomes

$$
K_{2}=\frac{\left\langle\Pi^{a}\right\rangle_{k, 0}}{V k}=\left.\frac{1}{V} \frac{\partial\left\langle\Pi^{a}\right\rangle_{k, 0}}{\partial k}\right|_{0,0},
$$

where we use $\left\langle\Pi^{a}\right\rangle_{0,0}=0$. We note that

$$
\left.\frac{\partial}{\partial \lambda}\left\langle\Pi^{a}\right\rangle_{k, \lambda}\right|_{0,0} \propto\left\langle\Pi^{a} \mathcal{U}^{c}\right\rangle_{0,0}=0,
$$

which confirms the assumption made earlier, that $K_{2}$ is independent of $\lambda$ at low $\lambda$. Dividing Eq. (30) by Eq. (31) and substituting $h_{\lambda}=K_{2} q_{\lambda}$ gives

$$
q^{\prime}=\frac{q_{\lambda}}{\lambda}=-\frac{\left\langle\Pi^{c}\right\rangle_{0,0}}{\left\langle\Pi^{a}\right\rangle_{k, 0} / k} .
$$

Equations (30)-(32), valid for weakly chiral systems, constitute the second main set of results of this paper. They show that the key properties may be derived from just two measurements made on the achiral reference system: the ensemble average $\left\langle\Pi^{c}\right\rangle_{0,0}$ of the chiral part of the torque per unit area, and the response of the achiral term to an applied twist, $\left\langle\Pi^{a}\right\rangle_{k, 0} / k$. Bearing in mind that $\left\langle\Pi^{c}\right\rangle_{k, 0}=\left\langle\Pi^{c}\right\rangle_{0,0}$ $+\mathcal{O}\left(k^{2}\right)$ (see the Appendix), both measurements may be made, if desired, in a single simulation in twisted boundaries.

Since Eqs. (30)-(32) involve simple sums of pairwise functions, they will give information, not only about systems 
in which all molecules are chiral, but also those in which just one molecule is chiral. If we define the pair average property, for an arbitrary pair 1 and 2,

$$
\left\langle\pi^{c}\right\rangle=-\left\langle r_{12 z} \tau_{12 z}^{c}\right\rangle,
$$

then for a system of $N$ (weakly) chiral molecules,

$$
h^{\prime}=-\frac{\frac{1}{2} N(N-1)}{V}\left\langle\pi^{c}\right\rangle_{0,0},
$$

while for a dilute system of $n$ chiral dopants, interacting with strength $\lambda$ with the $N$ achiral solvent molecules,

$$
h^{\prime}=-\frac{n N}{V}\left\langle\pi^{c}\right\rangle_{0,0}
$$

Note that, in this linear response regime, the coefficient may be expressed in terms of direct interactions between the dopant and the surroundings: perturbations of the liquid structure are secondary and would come in at higher order, as usual. ${ }^{6,7}$ Last, in this regime, the above equations may easily be written in terms of the pair distribution function and used to predict the chiral properties of a wide range of perturbed molecular structures.

\section{SIMULATION}

To test the new method, we chose a simple model potential for rigid chiral mesogenic particles that is a linear combination of an achiral and a chiral term, each of which is, as usual, pairwise additive:

$$
\mathcal{U}_{\lambda}=\mathcal{U}^{a}+\lambda \mathcal{U}^{c}=\sum_{i<j}\left(u_{i j}^{a}+\lambda u_{i j}^{c}\right)
$$

Each pair is described by the separation vector $\mathbf{r}_{i j}=\mathbf{r}_{i}-\mathbf{r}_{j}$ and the unit orientation vectors $\hat{\mathbf{e}}_{i}$ and $\hat{\mathbf{e}}_{j}$. The achiral part is the popular Gay-Berne potential, ${ }^{15}$

$$
\begin{aligned}
u_{i j}^{a} & =u_{i j}^{\mathrm{GB}}\left(\mathbf{r}_{i j}, \hat{\mathbf{e}}_{i}, \hat{\mathbf{e}}_{j}\right) \\
& =4 \epsilon\left(\hat{\mathbf{r}}_{i j}, \hat{\mathbf{e}}_{i}, \hat{\mathbf{e}}_{j}\right)\left[\varrho_{i j}^{-12}\left(\mathbf{r}_{i j}, \hat{\mathbf{e}}_{i}, \hat{\mathbf{e}}_{j}\right)-\varrho_{i j}^{-6}\left(\mathbf{r}_{i j}, \hat{\mathbf{e}}_{i}, \hat{\mathbf{e}}_{j}\right)\right],
\end{aligned}
$$

with reduced distance

$$
\varrho_{i j}\left(\mathbf{r}_{i j}, \hat{\mathbf{e}}_{i}, \hat{\mathbf{e}}_{j}\right)=\frac{r_{i j}-\sigma\left(\hat{\mathbf{r}}_{i j}, \hat{\mathbf{e}}_{i}, \hat{\mathbf{e}}_{j}\right)+\sigma_{0}}{\sigma_{0}},
$$

shape function

$$
\begin{aligned}
\sigma\left(\hat{\mathbf{r}}_{i j}, \hat{\mathbf{e}}_{i}, \hat{\mathbf{e}}_{j}\right)= & \sigma_{0}\left[1-\frac{\chi}{2}\left(\frac{\left(\hat{\mathbf{r}}_{i j} \cdot \hat{\mathbf{e}}_{i}+\hat{\mathbf{r}}_{i j} \cdot \hat{\mathbf{e}}_{j}\right)^{2}}{1+\chi \hat{\mathbf{e}}_{i} \cdot \hat{\mathbf{e}}_{j}}\right.\right. \\
& \left.\left.+\frac{\left(\hat{\mathbf{r}}_{i j} \cdot \hat{\mathbf{e}}_{i}-\hat{\mathbf{r}}_{i j} \cdot \hat{\mathbf{e}}_{j}\right)^{2}}{1-\chi \hat{\mathbf{e}}_{i} \cdot \hat{\mathbf{e}}_{j}}\right)\right]^{-1 / 2},
\end{aligned}
$$

and energy function

$$
\begin{aligned}
& \boldsymbol{\epsilon}\left(\hat{\mathbf{r}}_{i j}, \hat{\mathbf{e}}_{i}, \hat{\mathbf{e}}_{j}\right)=\boldsymbol{\epsilon}_{0}\left[\boldsymbol{\epsilon}_{1}\left(\hat{\mathbf{e}}_{i}, \hat{\mathbf{e}}_{j}\right)\right]^{\nu}\left[\boldsymbol{\epsilon}_{2}\left(\hat{\mathbf{r}}_{i j}, \hat{\mathbf{e}}_{i}, \hat{\mathbf{e}}_{j}\right)\right]^{\mu}, \\
& \boldsymbol{\epsilon}_{1}\left(\hat{\mathbf{e}}_{i}, \hat{\mathbf{e}}_{j}\right)=\left[1-\left(\chi \hat{\mathbf{e}}_{i} \cdot \hat{\mathbf{e}}_{j}\right)^{2}\right]^{-1 / 2},
\end{aligned}
$$

$$
\begin{aligned}
\boldsymbol{\epsilon}_{2}\left(\hat{\mathbf{r}}_{i j}, \hat{\mathbf{e}}_{i}, \hat{\mathbf{e}}_{j}\right)= & 1-\frac{\chi^{\prime}}{2}\left(\frac{\left(\hat{\mathbf{r}}_{i j} \cdot \hat{\mathbf{e}}_{i}+\hat{\mathbf{r}}_{i j} \cdot \hat{\mathbf{e}}_{j}\right)^{2}}{1+\chi^{\prime} \hat{\mathbf{e}}_{i} \cdot \hat{\mathbf{e}}_{j}}\right. \\
& \left.+\frac{\left(\hat{\mathbf{r}}_{i j} \cdot \hat{\mathbf{e}}_{i}-\hat{\mathbf{r}}_{i j} \cdot \hat{\mathbf{e}}_{j}\right)^{2}}{1-\chi^{\prime} \hat{\mathbf{e}}_{i} \cdot \hat{\mathbf{e}}_{j}}\right) .
\end{aligned}
$$

In the above equations $\hat{\mathbf{r}}_{i j}=\mathbf{r}_{i j} / r_{i j}$. The shape function is determined by a shape anisotropy parameter,

$$
\chi=\frac{\kappa^{2}-1}{\kappa^{2}+1}, \quad \kappa=\frac{\sigma_{\text {end-end }}}{\sigma_{\text {side-side }}},
$$

and the energy function by a well-depth anisotropy parameter,

$$
\chi^{\prime}=\frac{\kappa^{\prime 1 / \mu}-1}{\kappa^{\prime 1 / \mu}+1}, \quad \kappa^{\prime}=\frac{\epsilon_{\text {side-side }}}{\epsilon_{\text {end-end }}} .
$$

In Eqs. (37)-(41) we used the Berardi-Zannoni ${ }^{16}$ parameter values $\mu=1, \nu=3, \kappa=3, \kappa^{\prime}=5$. Henceforth, distance and energy units are defined so that in Eqs. (35)-(37), $\sigma_{0}=\epsilon_{0}$ $=1$.

For the chiral part of the potential we used the form proposed by Memmer and Kuball, ${ }^{17}$

$$
u_{i j}^{c}=u_{i j}^{\mathrm{MK}}\left(\mathbf{r}_{i j}, \hat{\mathbf{e}}_{i}, \hat{\mathbf{e}}_{j}\right)=4 \epsilon \varrho_{i j}^{-7}\left(\hat{\mathbf{e}}_{i} \times \hat{\mathbf{e}}_{j} \cdot \hat{\mathbf{r}}_{i j}\right)\left(\hat{\mathbf{e}}_{i} \cdot \hat{\mathbf{e}}_{j}\right),
$$

where $\epsilon$ and $\varrho_{i j}$ are the same functions appearing in the Gay-Berne potential. The combination $\left(\hat{\mathbf{e}}_{i} \times \hat{\mathbf{e}}_{j} \cdot \hat{\mathbf{r}}_{i j}\right)\left(\hat{\mathbf{e}}_{i} \cdot \hat{\mathbf{e}}_{j}\right)$ is the simplest pseudoscalar and head-tail symmetric expression that can be formed with $\hat{\mathbf{r}}_{i j}, \hat{\mathbf{e}}_{i}, \hat{\mathbf{e}}_{j}$. The exponent of the separation-dependent term $\varrho_{i j}^{-7}$ has the asymptotic $r_{i j}^{-7}$ form of the multipole expansion of the chiral interaction energy arising from quantum mechanical fluctuations of the electron distribution (dispersion interactions). ${ }^{17-19}$ It has been noted $^{8-10}$ that the leading term in a multipole expansion of an interaction potential based simply on summing site-site interactions must have a higher-order dependence on orientations than the $\left(\hat{\mathbf{e}}_{i} \times \hat{\mathbf{e}}_{j} \cdot \hat{\mathbf{r}}_{i j}\right)\left(\hat{\mathbf{e}}_{i} \cdot \hat{\mathbf{e}}_{j}\right)$ term considered here: in this sense it is an idealized model.

Both parts of the pair potential were cut at the distance $r_{\text {cut }}=4 \sigma_{0}$ and shifted by an orientation-dependent quantity:

$$
\begin{aligned}
& u_{i j}^{a}\left(\mathbf{r}_{i j}, \hat{\mathbf{e}}_{i}, \hat{\mathbf{e}}_{j}\right) \\
& \quad= \begin{cases}u_{i j}^{\mathrm{GB}}\left(\mathbf{r}_{i j}, \hat{\mathbf{e}}_{i}, \hat{\mathbf{e}}_{j}\right)-u_{i j}^{\mathrm{GB}}\left(\hat{\mathbf{r}}_{i j} r_{\mathrm{cut}}, \hat{\mathbf{e}}_{i}, \hat{\mathbf{e}}_{j}\right), \quad r_{i j}<r_{\mathrm{cut}}, \\
0, \quad r_{i j} \geqslant r_{\mathrm{cut}},\end{cases}
\end{aligned}
$$

a similar equation connects $u_{i j}^{c}$ with $u_{i j}^{\mathrm{MK}}$.

We conducted standard canonical ensemble Monte Carlo simulations ${ }^{20}$ using $N=1024$ or 2048 particles in a rectangular box with side ratios $L_{x}: L_{y}: L_{z}=1: 1: 2$ or $1: 1: 4$, respectively, and a number density of 0.3 (in the reduced units defined earlier). A snapshot of the 1024-particle system in twisted boundary conditions is given in Fig. 1. To help the comparison with twisted configurations where the director rotates in a plane perpendicular to the twist axis $z$, in conventional, untwisted PBCs when $k=0$ we constrained the director to the same $x y$ plane by adding a term proportional to $\left(Q_{x z}^{2}+Q_{y z}^{2}\right)$ to the energy $\mathcal{U}_{\lambda}$ defined in Eq. (33); $\mathbf{Q}$ is the order tensor: 


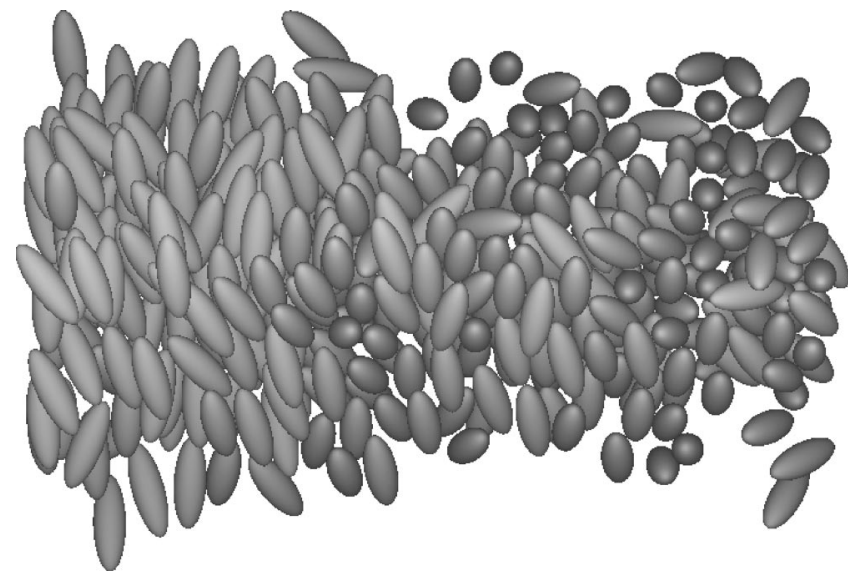

FIG. 1. Snapshot of the model system with $N=1024$ at $T=2.8, \lambda=0.01$, and $k L_{z}=\pi / 2$ (twisted PBCs).

$$
Q_{\alpha \beta}=\frac{1}{N} \sum_{i=1}^{N}\left(\frac{3}{2} \hat{e}_{i \alpha} \hat{e}_{i \beta}-\frac{1}{2} \delta_{\alpha \beta}\right) .
$$

We averaged $300(N=1024)$ or $150(N=2048)$ blocks of 2000 attempted moves per particle for each data point; we verified that the block size is sufficiently large that the block averages are independent from each other, and the run lengths such that the relative errors in simulation averages are about $1 \%$; the maximum particle move displacement and rotation were both set to 0.1 to give an acceptance ratio of $50 \%$. These calculations yielded $K_{2} q_{\lambda}$ from thermodynamic integration, Eq. (4), and from our new method, Eq. (12) or, in the special case of weak chirality, Eq. (30).

To have an independent estimate of $K_{2}$, that our new method gives separately from $q_{\lambda}$, Eq. (14) or, for weak chirality, Eq. (31), we accessed $K_{2}$ through orientational order fluctuations, exploiting the fact ${ }^{21}$ that in a 123 axis system where $\langle\mathbf{Q}\rangle$ is diagonal with $\hat{\mathbf{n}}=(0,0,1)$, if a wave vector $\mathbf{k}$ is chosen such that $k_{2}=0$, then for $k \rightarrow 0$

$$
\widetilde{W}_{a 3}\left(k_{1}, k_{3}\right)=\frac{9 P_{2}^{2} V k_{\mathrm{B}} T}{4\left\langle\left|\widetilde{Q}_{a 3}(\mathbf{k})\right|^{2}\right\rangle} \sim K_{a} k_{1}^{2}+K_{3} k_{3}^{2},
$$

with $a=1,2$. In this equation, $P_{2}$ is the nematic order parameter, defined as the highest eigenvalue of $\langle\mathbf{Q}\rangle$, and $\widetilde{\mathbf{Q}}(\mathbf{k})$ is the order tensor in reciprocal space:

$$
\widetilde{Q}_{\alpha \beta}(\mathbf{k})=\frac{V}{N} \sum_{i=1}^{N}\left(\frac{3}{2} \hat{e}_{i \alpha} \hat{e}_{i \beta}-\frac{1}{2} \delta_{\alpha \beta}\right) \exp \left(i \mathbf{k} \cdot \mathbf{r}_{i}\right) .
$$

For this calculation an extrapolation to $k=0$ is required: accordingly, we employed a large system of $N=16000 \mathrm{~mol}-$ ecules, and used a domain decomposition parallel molecular dynamics program. The molecular mass and moment of inertia were chosen to be unity, and in the reduced units of time so defined, a time step $\delta t=0.003$ was found to give satisfactory energy conservation, using the RATTLE integration algorithm. ${ }^{22}$ A simulation run length of $10^{6}$ steps gave adequate sampling of the lowest-frequency modes. To facilitate the analysis, the director-based 123 frame was fixed in coincidence with the $x y z$ frame of the simulation box by adding two extra Lagrange multipliers to the dynamical

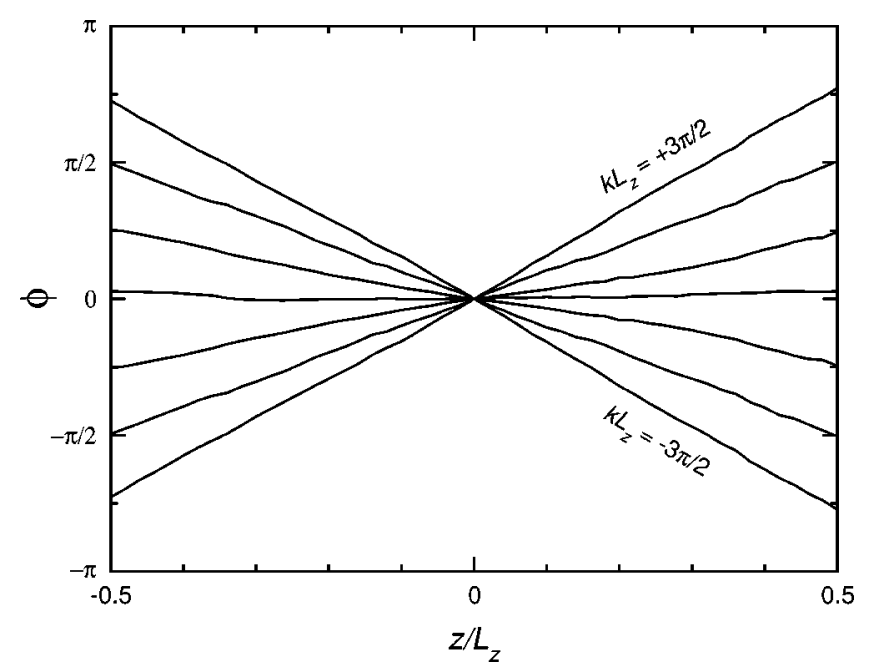

FIG. 2. The angle $\phi$ as a function of $z / L_{z}$, Eq. (2), for different metastable states of the system with $N=1024$ and $T=2.80$. An arbitrary phase factor has been added so that $\phi(0)=0$.

equations so that $Q_{x z}=Q_{y z}=0$ at every time step. ${ }^{23}$ We used an elongated box with side ratios $L_{x}: L_{y}: L_{z}=1: 1: 2$. The k-dependent simulation averages required in Eq. (45) were sampled on a $6 \times 6 \times 12$ grid of points in the $x y z$ frame, and the resulting $\widetilde{W}_{a 3}$ surfaces were fitted with fourth-degree polynomial functions of $k_{1}^{2}$ and $k_{3}^{2}$ constrained to pass through the origin $\widetilde{W}_{a 3}(0,0)=0$. The fourth-order coefficients in the fits were very small, and the first-order coefficients gave the desired elastic constants. Apart from the improved, symplectic integrator with global Lagrange constraints on $\mathbf{Q}$, we followed closely the procedure of Ref. 24.

\section{RESULTS}

With our Monte Carlo program we investigated thoroughly the temperature $T=2.8$ with $N=1024$ and values of chiral strength $|\lambda| \leqslant 1.5$. Although it has to be kept in mind that only values of $|\lambda| \approx 0.01$ make physical sense if the particles are to be interpreted as molecules, we were interested in exploring the whole range, where a uniform twisted nematic phase satisfying Eq. (2) is (at least meta-) stable; we found this to be true for values $|\lambda|<1$. In choosing values of $k$ and $\lambda$ we took advantage of the relations given in the Appendix to symmetrize our results. Typical director angle profiles $\phi(z)$ are shown in Fig. 2; the chiral part of the energy $\left\langle\mathcal{U}^{c}\right\rangle_{k, \lambda}$ and the torque per unit area $\left\langle\Pi_{\lambda}\right\rangle_{k, \lambda}$ are plotted as functions of chirality strength $\lambda$ in Figs. 3 and 4, respectively. For any given value of $\lambda$, we typically find one director configuration of well-defined wave number $k$ that is stable in each of the two boundary conditions (untwisted and twisted). An exception is the low-chirality range $-0.2 \leqslant \lambda$ $\leqslant 0.20$ for which we observed three stable states: $k L_{z}=0$ and $k L_{z}= \pm \pi / 2$. On increasing the chirality we observed spontaneous jumps in $k L_{z}: 0 \rightarrow \pi$ at $\lambda \approx 0.5, \pi / 2 \rightarrow 3 \pi / 2$ at $\lambda$ $=0.7$, with symmetrical jumps in the negative $\lambda$ direction. The twist remains uniform until about $\lambda \simeq 1$; beyond this point progressive deviations from a linear $\phi(z)$ profile arise. This may correspond to mesophases other than the twisted 


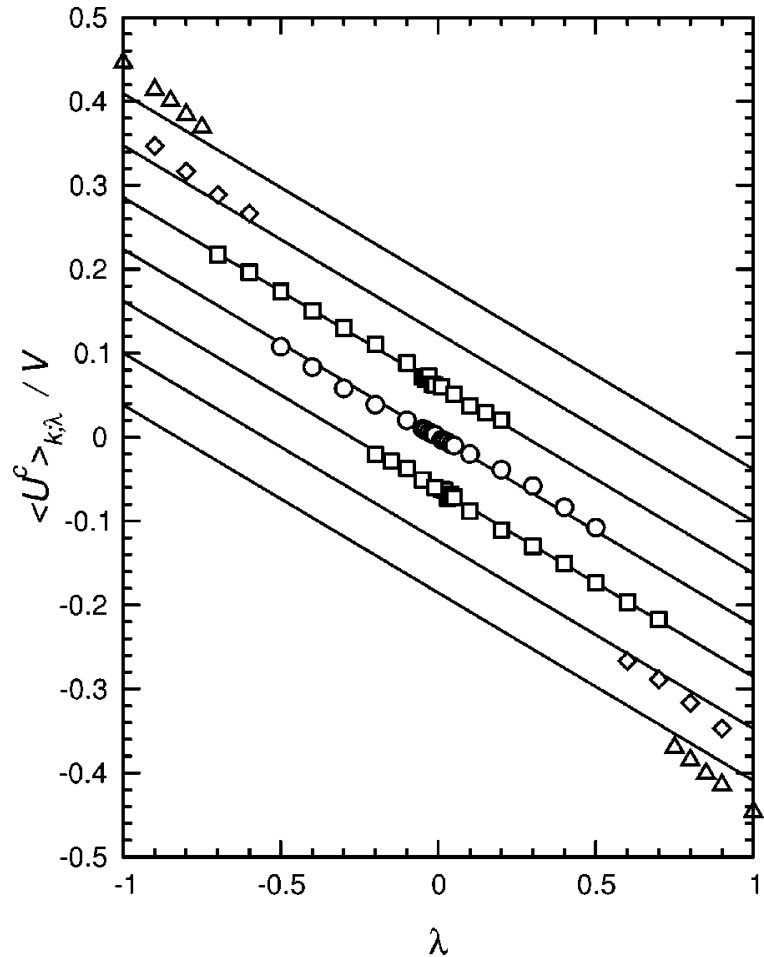

FIG. 3. Chiral part of the internal energy density $\left\langle\mathcal{U}^{c}\right\rangle_{k, \lambda} / V$ as a function of the chiral strength $\lambda$, at various twist wave numbers $k$ for the system with $N=1024$ and $T=2.8$. Where the twist is uniform the dependence is linear. Circles: $k L_{z}=0$. Squares: $k L_{z}= \pm \pi / 2$. Diamonds: $k L_{z}= \pm \pi$. Triangles: $k L_{z}= \pm 3 \pi / 2$. Positive values of $k$ correspond to positive $\lambda$, and vice versa. The slope of the full lines (one for each $k$ value) and the spacing among them are determined by a linear fit, Eq. (23), to the $k L_{z}=0$ and $k L_{z}=\pi / 2$ results, which determine $K_{2} q_{\lambda}$ from thermodynamic integration, Eq. (48). Error bars have been omitted in this and the following figures because they are about the same size as the symbols used for the data points.

nematic, that have been described elsewhere. ${ }^{17}$ We were not interested in studying them further, because the method we are presenting here is based on a uniformly twisted director, Eq. (2).

For constant wave number $k$, and over the range where $\phi(z)$ is linear, $\left\langle\mathcal{U}^{c}\right\rangle_{k, \lambda}$ varies linearly with $\lambda$; this is in agreement with the prediction of liner response theory, Eq. (23). $\left\langle\Pi_{\lambda}\right\rangle_{k, \lambda}$ also varies linearly with $\lambda$. In fact, the torque measurements conform to the behavior discussed in connection with Eqs. (30)-(32), and in the Appendix: we see that $\left\langle\Pi^{c}\right\rangle_{k, \lambda}$ is essentially independent of both $\lambda$ and $k$ for all of our simulations, while $\left\langle\Pi^{a}\right\rangle_{0,0}$ vanishes, and $\left\langle\Pi^{a}\right\rangle_{k, \lambda}$ is independent of $\lambda$ for fixed $k$. Therefore, the linear variation of $\left\langle\Pi_{\lambda}\right\rangle_{k, \lambda}$ with $\lambda$ at fixed $k$ arises simply through the explicit factor $\lambda$ in the definition (20).

These simplifying features make the computation of the integral on the left side of Eq. (5) particularly easy: fitting a line, Eq. (23), to $\left\langle\mathcal{U}^{c}\right\rangle_{k, \lambda}$ in the interval $|\lambda| \leqslant 0.7$ for $k L_{z}$ $=\pi / 2$ yields simply

$$
\Delta \mathcal{F}_{k, \pm \lambda}=2 \lambda\left\langle\mathcal{U}^{c}\right\rangle_{k, 0},
$$

and inserting this into Eq. (4),

$$
h^{\prime}=\frac{K_{2} q_{\lambda}}{\lambda}=-\frac{\left\langle\mathcal{U}^{c}\right\rangle_{k, 0}}{V k}
$$

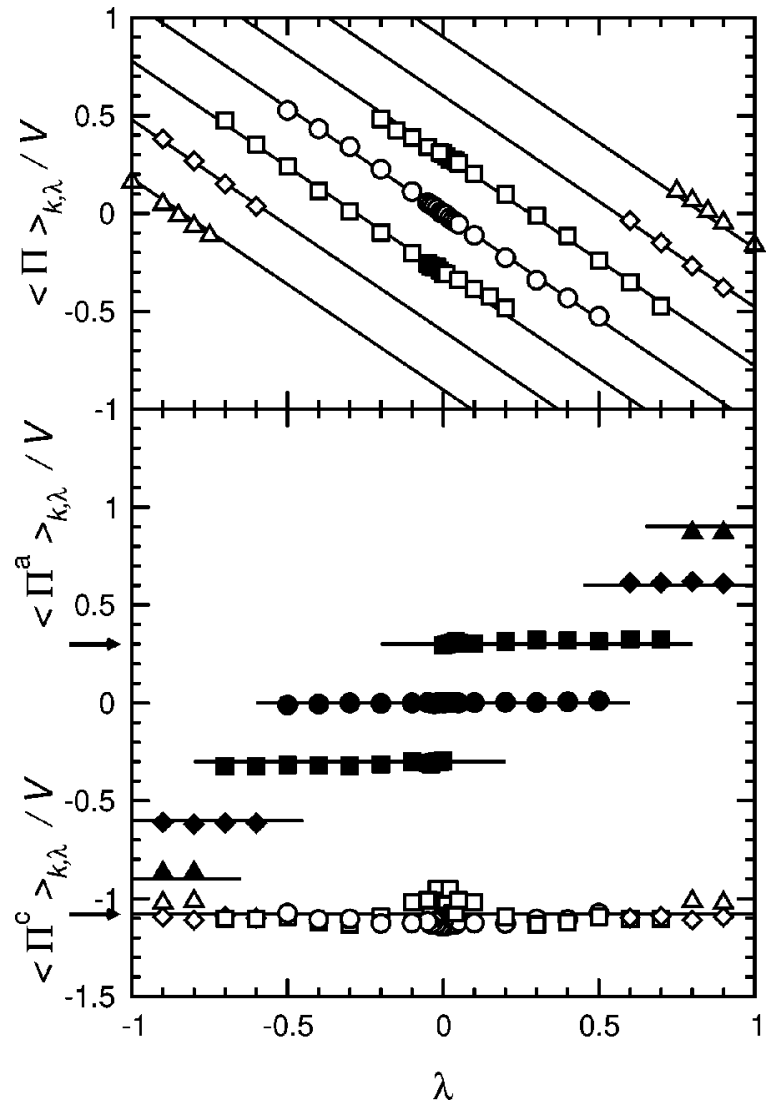

FIG. 4. Torque per unit area $\left\langle\Pi_{\lambda}\right\rangle_{k, \lambda} / V$, and components $\left\langle\Pi^{a}\right\rangle_{k, \lambda} / V$ (full symbols) and $\left\langle\Pi^{c}\right\rangle_{k, \lambda} / V$ as functions of the chiral strength $\lambda$, at various twist wave numbers, for the system with $N=1024$ and $T=2.80$. Where the twist is uniform the dependence is linear. The slope of the full lines in the uppermost graph (one for each $k$ value) gives $h^{\prime}$, Eq. (28), and hence $h_{\lambda}$ $=K_{2} q_{\lambda}$, Eq. (12); the distance between them gives $K_{2}$, Eq. (14). Arrows indicate the two values $\left\langle\Pi^{c}\right\rangle_{k, 0} / V$ (with $k=0$ or $k \neq 0$ ) and $\left\langle\Pi^{a}\right\rangle_{k, 0} / V$ (with $k \neq 0$ ), which in the case of weak chirality may be measured in the achiral system to yield the same quantities through Eqs. (30) and (31). The notation is the same as for Fig. 3.

also see Eq. (26). The results are reported in Table I together with the results from the torque measurement according to Eq. (28); see also Fig. 4. Moreover, Table I shows the value of $K_{2}$ from the torque measurement, Eq. (14), and from order fluctuation analysis, Eq. (45). Note in Fig. 4 that $\left\langle\Pi_{\lambda}\right\rangle_{k, \lambda}$ $-\left\langle\Pi_{\lambda}\right\rangle_{0, \lambda}$, and thus $K_{2}$, is independent of $\lambda$; the order fluctuation calculation was done with $\lambda=0$.

TABLE I. A comparison between the new torque-measurement method and two reference methods: thermodynamic integration (for $h^{\prime}=K_{2} q_{\lambda} / \lambda$ ) and order fluctuation analysis (for $K_{2}$ ); estimated statistical errors in the last quoted digits are given in parentheses. At the two higher temperatures we could not observe a uniformly twisted director according to Eq. (2), and thus our analysis was applicable only for $k=0$.

\begin{tabular}{lcccccc}
\hline \hline & & \multicolumn{2}{c}{$h^{\prime}$} & & \multicolumn{2}{c}{$K_{2}$} \\
\cline { 3 - 4 } \cline { 6 - 7 }$T$ & $N$ & $\begin{array}{c}\text { Torque } \\
\text { Eq. (28) }\end{array}$ & $\begin{array}{c}\text { Integration } \\
\text { Eq. (4) }\end{array}$ & & $\begin{array}{c}\text { Torque } \\
\text { Eq. (14) }\end{array}$ & $\begin{array}{c}\text { Fluctuation } \\
\text { Eq. (45) }\end{array}$ \\
\hline 2.80 & 1024 & $1.08(1)$ & $0.94(2)$ & & $4.55(2)$ & $4.54(11)$ \\
2.80 & 2048 & $1.08(1)$ & $1.08(2)$ & & $4.68(3)$ & \\
3.40 & 1024 & $0.63(1)$ & $\cdots$ & & $\cdots$ & $1.98(5)$ \\
3.45 & 1024 & $0.56(1)$ & $\cdots$ & & $\cdots$ & $1.59(4)$ \\
\hline \hline
\end{tabular}


The agreement between the torque measurement and the thermodynamic integration measurement of $h^{\prime}$ is much better for the 1:1:4 box of the $N=2048$ system than for the 1:1:2 box of the $N=1024$ system. This is most likely a finitesize effect due to the higher value of $k$ in the smaller box, affecting the thermodynamic integration value, because $h^{\prime}$ is found by the torque measurement with $k=0$. The agreement between different measurements of $K_{2}$ is within the sum of the estimated standard deviations; by chance, it is better in the smaller system.

We repeated the above calculations at $T=3.40$ and $T$ $=3.45$ varying $\lambda$ in a narrower range $|\lambda| \leqslant 0.05$ at intervals of 0.01 . The choice of these temperatures was motivated by the availability in the literature of reference values for $K_{2}$ from order fluctuations ${ }^{24}$ though we ended up recalculating them with our improved director constraint algorithm mentioned above, that we developed to compute the reference value for $K_{2}$ at $T=2.80$. Our new fluctuation values for $K_{2}$ at $T=3.40$ and $T=3.45$ are $15 \%$ higher than the old ones. Unfortunately at these temperatures we could not observe a uniformly twisted director according to Eq. (2), and thus our analysis was applicable only for $k=0$, i.e., we could calculate only $h^{\prime}$; the values are reported in Table I. We believe that the director nonuniformity at these higher temperatures is due to the proximity of the nematic-isotropic transition temperature, here $T=3.57:{ }^{16}$ the free energy barriers around the metastable states are lower and can be crossed more easily. Indeed we observed the helix switching back and forth between its two possible senses of rotation. Although it restricts our calculations, this switching is an interesting phenomenon that has received theoretical attention recently, ${ }^{25}$ and here we confirm that it can be observed with computer simulation.

\section{DISCUSSION}

We have shown how the equilibrium pitch $q_{\lambda}$ of chiral nematics can be found by computer simulation sampling the torque per unit area $\Pi_{\lambda}$ in twisted and untwisted director configurations, as an alternative to thermodynamic integration; moreover, the $K_{2}$ elastic constant is a byproduct of the calculation instead of a required input parameter to be found by another method like an analysis of the order fluctuations ${ }^{24}$ or of the direct correlation function. ${ }^{26-30}$ The route to $q_{\lambda}$ and $K_{2}$ through $\left\langle\Pi_{\lambda}\right\rangle$ is more convenient than with thermodynamic integration or order fluctuation because the data analysis is easier and the computational effort smaller. With respect to thermodynamic integration there is the additional advantage that the simulation giving $h_{\lambda}=K_{2} q_{\lambda}$ can be done with $k=0$, and thus in a fairly small box; we have confirmed the practicality of the approach proposed in Refs. 8-10. Of course, the separate determination of $K_{2}$ by this method requires an additional simulation at $k \neq 0$, as suggested in Ref. 2.

In the linear response regime, for weak chirality, we have demonstrated the possibility of calculating the helicity parameter $h^{\prime}=K_{2} q_{\lambda} / \lambda$ directly from simulations in the untwisted and achiral reference system: this allows one to obtain results for a range of weakly chiral systems from simulations of a single reference system, and to isolate the features in the molecular interactions and pair structure responsible for chirality. If a simulation in twisted boundaries is performed, for example, to compute $K_{2}$, then the same simulation may be used to calculate $h^{\prime}$ at the same time, since this quantity does not vary (to first order) with a twist. However, we should emphasize again that the torque measurements are not restricted to the weakly chiral limit, nor do they require one to separate the interaction potential into chiral and achiral parts. Our prototype simulations can be repeated with realistic models of actual mesogens to compare with experimental measurements, at the price of a larger computational effort, if good force field parameters are available.

\section{ACKNOWLEDGMENTS}

This work was supported by EPSRC, the British Council through the ARC program, and the Leverhulme Foundation. Part of this work was carried out while G.G. and M.P.A. were at the H. H. Wills Physics Laboratory, University of Bristol. Part of the work was carried out while M.P.A. was on study leave at the Max Planck Institute for Polymer Research, Mainz, and the Institute for Physics, University of Mainz: the support of the Alexander von Humboldt Foundation through a Research Fellowship, and the hospitality of K. Kremer and K. Binder are gratefully acknowledged. Monte Carlo simulations were run on DEC Alpha workstations, an SGI Origin 2000 provided by the Joint Research Equipment Initiative, and a Beowulf PC cluster at Bristol; and on a SuSE SALT PC cluster and Compaq workstations at Bielefeld. Molecular dynamics simulations were run on the Edinburgh Cray T3E, using the code GBMEGA of the Complex Fluids Consortium. The authors acknowledge a helpful conversation with M. A. Osipov.

\section{APPENDIX A: SYMMETRY PROPERTIES}

Here we derive some symmetry relations that simplify the analysis in the main text. We assume that the potential energy may be written in the form of Eq. (19), and the torque per unit area as in Eq. (20):

$$
\begin{aligned}
& \mathcal{U}_{\lambda}(\mathbf{r}, \mathbf{e})=\mathcal{U}^{a}(\mathbf{r}, \mathbf{e})+\lambda \mathcal{U}^{c}(\mathbf{r}, \mathbf{e}), \\
& \Pi_{\lambda}(\mathbf{r}, \mathbf{e})=\Pi^{a}(\mathbf{r}, \mathbf{e})+\lambda \Pi^{c}(\mathbf{r}, \mathbf{e}),
\end{aligned}
$$

where $\mathcal{U}^{a}, \mathcal{U}^{c}, \Pi^{a}$, and $\Pi^{c}$ are all independent of the chirality parameter $\lambda$; we have abbreviated all molecular coordinates and orientation vectors as $\mathbf{r}, \mathbf{e}$, respectively. Symmetry with respect to inversion $\mathbf{r} \rightarrow-\mathbf{r}, \mathbf{e} \rightarrow-\mathbf{e}, \lambda \rightarrow-\lambda$ implies

$$
\begin{aligned}
& \mathcal{U}_{-\lambda}(-\mathbf{r},-\mathbf{e})=\mathcal{U}_{\lambda}(\mathbf{r}, \mathbf{e}), \quad \Pi_{-\lambda}(-\mathbf{r},-\mathbf{e})=-\Pi_{\lambda}(\mathbf{r}, \mathbf{e}), \\
& \mathcal{U}^{a}(-\mathbf{r},-\mathbf{e})=\mathcal{U}^{a}(\mathbf{r}, \mathbf{e}), \quad \Pi^{a}(-\mathbf{r},-\mathbf{e})=-\Pi^{a}(\mathbf{r}, \mathbf{e}), \\
& \mathcal{U}^{c}(-\mathbf{r},-\mathbf{e})=-\mathcal{U}^{c}(\mathbf{r}, \mathbf{e}), \quad \Pi^{c}(-\mathbf{r},-\mathbf{e})=\Pi^{c}(\mathbf{r}, \mathbf{e}) .
\end{aligned}
$$

The achiral Gay-Berne potential and the chiral MemmerKuball potential are easily shown to satisfy the above equations. Symmetry dictates that the canonical ensemble distribution function $\varrho_{k, \lambda}(\mathbf{r}, \mathbf{e})$ satisfies

$$
\varrho_{-k,-\lambda}(-\mathbf{r},-\mathbf{e})=\varrho_{k, \lambda}(\mathbf{r}, \mathbf{e}),
$$


and this allows the following relations to be straightforwardly derived:

$$
\begin{aligned}
& \left\langle\mathcal{U}_{-\lambda}\right\rangle_{-k,-\lambda}=\left\langle\mathcal{U}_{\lambda}\right\rangle_{k, \lambda}, \quad\left\langle\Pi_{-\lambda}\right\rangle_{-k,-\lambda}=-\left\langle\Pi_{\lambda}\right\rangle_{k, \lambda}, \\
& \left\langle\mathcal{U}^{a}\right\rangle_{-k,-\lambda}=\left\langle\mathcal{U}^{a}\right\rangle_{k, \lambda}, \quad\left\langle\Pi^{a}\right\rangle_{-k,-\lambda}=-\left\langle\Pi^{a}\right\rangle_{k, \lambda}, \\
& \left\langle\mathcal{U}^{c}\right\rangle_{-k,-\lambda}=-\left\langle\mathcal{U}^{c}\right\rangle_{k, \lambda}, \quad\left\langle\Pi^{c}\right\rangle_{-k,-\lambda}=\left\langle\Pi^{c}\right\rangle_{k, \lambda} .
\end{aligned}
$$

Hence, the $k \rightarrow 0, \lambda \rightarrow 0$ limits of $\left\langle\mathcal{U}^{c}\right\rangle$ and $\left\langle\Pi^{a}\right\rangle$ vanish:

$$
\left\langle\mathcal{U}^{c}\right\rangle_{0,0}=0, \quad\left\langle\Pi^{a}\right\rangle_{0,0}=0 .
$$

The corresponding limiting values of $\left\langle\mathcal{U}^{a}\right\rangle_{0,0}$ and $\left\langle\Pi^{c}\right\rangle_{0,0}$ do not vanish, but the above symmetry relations dictate that their gradients with respect to $k$ and $\lambda$ are zero:

$$
\begin{aligned}
& \left.\frac{\partial}{\partial k}\left\langle\mathcal{U}^{a}\right\rangle_{k, \lambda}\right|_{0,0}=\left.\frac{\partial}{\partial \lambda}\left\langle\mathcal{U}^{a}\right\rangle_{k, \lambda}\right|_{0,0}=0, \\
& \left.\frac{\partial}{\partial k}\left\langle\Pi^{c}\right\rangle_{k, \lambda}\right|_{0,0}=\left.\frac{\partial}{\partial \lambda}\left\langle\Pi^{c}\right\rangle_{k, \lambda}\right|_{0,0}=0 .
\end{aligned}
$$

The corresponding thermodynamic linear response coefficients vanish, for instance,

$$
\left.\frac{\partial}{\partial \lambda}\left\langle\Pi^{c}\right\rangle_{k, \lambda}\right|_{0,0}=0 \Leftrightarrow\left\langle\Pi^{c} \mathcal{U}^{c}\right\rangle_{0,0}=0 .
$$

${ }^{1}$ P. G. de Gennes and J. Prost, The Physics of Liquid Crystals (Clarendon, Oxford, 1995).

${ }^{2}$ M. P. Allen and A. J. Masters, Mol. Phys. 79, 277 (1993).

${ }^{3}$ M. P. Allen, Phys. Rev. E 47, 4611 (1993).

${ }^{4}$ P. J. Camp, Mol. Phys. 91, 381 (1997).

${ }^{5}$ M. J. Cook and M. R. Wilson, J. Chem. Phys. 112, 1560 (2000).

${ }^{6}$ C. G. Gray and K. E. Gubbins, Theory of Molecular Fluids. Vol. 1: Fundamentals (Clarendon, Oxford, 1984)
${ }^{7}$ J.-P. Hansen and I. R. McDonald, Theory of Simple Liquids, 2nd ed. (Academic, London, 1986).

${ }^{8}$ A. B. Harris, R. D. Kamien, and T. C. Lubensky, Phys. Rev. Lett. 78, 1476 (1997)

${ }^{9}$ A. B. Harris, R. D. Kamien, and T. C. Lubensky, Phys. Rev. Lett. 78, 2867 (1997)

${ }^{10}$ A. B. Harris, R. D. Kamien, and T. C. Lubensky, Rev. Mod. Phys. 71, 1745 (1999)

${ }^{11}$ V. B. Nemtsov and M. A. Osipov, Sov. Phys. Crystallogr. 31, 125 (1986).

${ }^{12}$ J. L. Billeter and R. A. Pelcovits, Liq. Cryst. 27, 1151 (2000).

${ }^{13}$ J. M. Haile, Molecular Dynamics Simulation: Elementary Methods (Wiley, New York, 1992).

${ }^{14}$ M. P. Allen and A. J. Masters, J. Mater. Sci. 11, 2678 (2001).

${ }^{15}$ J. G. Gay and B. J. Berne, J. Chem. Phys. 74, 3316 (1981).

${ }^{16}$ R. Berardi, A. P. J. Emerson, and C. Zannoni, J. Chem. Soc., Faraday Trans. 89, 4069 (1993).

${ }^{17}$ R. Memmer, H. G. Kuball, and A. Schönhofer, Liq. Cryst. 15, 345 (1993).

${ }^{18}$ W. J. A. Goossens, Mol. Cryst. Liq. Cryst. 12, 237 (1971).

${ }^{19}$ B. W. van der Meer, G. Vertogen, A. J. Dekker, and J. G. J. Ypma, J. Chem. Phys. 65, 3935 (1976).

${ }^{20}$ M. P. Allen and D. J. Tildesley, Computer Simulation of Liquids, paperback ed. (Clarendon, Oxford, 1989).

${ }^{21}$ D. Forster, "Hydrodynamic fluctuations, broken symmetry and correlation functions," Frontiers in Physics (Benjamin, Reading, 1975), Vol. 47.

${ }^{22}$ H. C. Andersen, J. Comput. Phys. 52, 24 (1983).

${ }^{23}$ G. Germano (unpublished).

${ }^{24}$ M. P. Allen, M. A. Warren, M. R. Wilson, A. Sauron, and W. Smith, J. Chem. Phys. 105, 2850 (1996).

${ }^{25}$ A. V. Emelyanenko, M. A. Osipov, and D. A. Dunmur, Phys. Rev. E 62, 2340 (2000).

${ }^{26}$ J. Stelzer, L. Longa, and H. R. Trebin, Mol. Cryst. Liq. Cryst. Sci. Technol., Sect. A 262, 455 (1995).

${ }^{27}$ J. Stelzer, H.-R. Trebin, and L. Longa, J. Chem. Phys. 107, 1295 (1997) (E).

${ }^{28}$ A. V. Zakharov and A. Maliniak, Eur. Phys. J. E 4, 85 (2001).

${ }^{29}$ N. H. Phuong, G. Germano, and F. Schmid, Comput. Phys. Commun. (in press).

${ }^{30}$ N. H. Phuong, G. Germano, and F. Schmid, J. Chem. Phys. 115, 7227 (2001) 